\title{
WAVE ENERGY CONVERSION USING A BLOW-JET SYSTEM
}

\author{
Edgar Mendoza-Baldwin¹, Rodolfo Silva-Casarín ${ }^{1}$ Rafael Sánchez-Dirzo ${ }^{1}$ and Xavier \\ Chávez-Cárdenas ${ }^{1}$
}

\begin{abstract}
This paper presents the results of exhaustive experimental work focused on evaluating the efficiency of two devices as wave energy converters and as coastal protection alternatives. The first device is a wave amplifier that by means of overtopping stores water in a reservoir where potential energy can be used to produce power. The second device, the Blow-Jet, is a novel proposal that gathers together the operation of a tapchan and a blowhole to generate an intermittent jet that can easily feed a turbine. Results show that for both devices there is a strong dependency on the wave climate but that there is a possibility of optimizing geometric designs. Transmission coefficient values obtained for the Blow-Jet point to a real chance for its use as a multi-purpose coastal structure.
\end{abstract}

Keywords: wave energy conversion; tapchan; blow-jet; shore protection

\section{INTRODUCTION}

The search for new sources of clean, renewable energy has led an increasing number of scientists to look towards wave energy conversion. The main advantage of wave energy is that it has no fuel costs; its disadvantage is the oscillatory nature of waves (Leijon et al, 2006). Estimations carried out by the International Energy Agency suggest that wave energy could contribute between 10 and $50 \%$ of annual electricity demand worldwide. Gravity waves carry clean and sustainable energy that can be captured by Wave Energy Converters (WEC) placed near shorelines or in deep water. Several types of WEC have been designed and these can be classified in two general categories: a) fixed generating devices, e.g. oscillating water columns (OWC), tapered channel systems (Tapchan) and the waveroller; and b) floating generating devices, e.g. Pelamis, Salter Duck, Wave Dragon, Archimedes Wave Swing (AWS), Mighty Whale, PowerBuoys, the CETO system and the Energetech system (IEA-OES, 2008).

On the other hand, there is an undeniable, worldwide need for coastal protection. It is well known that around $70 \%$ of the world's sand beaches are being eroded (Hsu et al, 2005) and the evidence of sea level rise (considering the effects of changing climate or not) are aggravating that problem. As an example of sea level rise, Figure 1 (Uriarte, 2003) shows some very simple time series for three locations at Pacific Ocean.

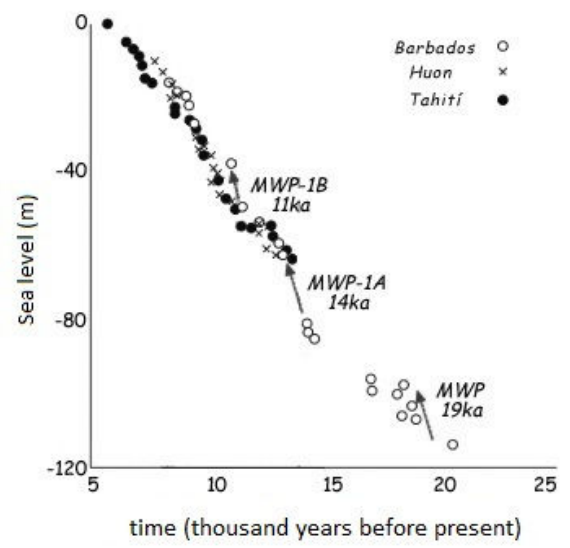

Figure 1. Time series of sea level rise (Uriarte, 2003).

The aforementioned objectives are the motivation behind the research taking place at the National University of Mexico (UNAM). The main thrust of the work is to find designs suitable as multipurpose structures, in other words, designing coastal infrastructure which is capable of fulfilling more than one littoral demand. In the specific case presented in this paper, the structure designed and evaluated should be acceptable as a wave energy converter as well as providing coastal protection. In Mexico, this kind of solution is encouraged, being designed according to local, natural cycles and being environmentally friendly. Small, submerged structures are preferable to more aggressive types because of the relevance of tourism on Mexican coasts. An important part of the motivation for this work comes

\footnotetext{
${ }^{1}$ Engineering Institute, National University of Mexico, Ciudad Universitaria, Mexico City, 04360, México
} 
from the fact that over recent years several Mexican sand coasts have been dramatically eroded due to human activities combined with extreme meteorological events (see Figure 2).

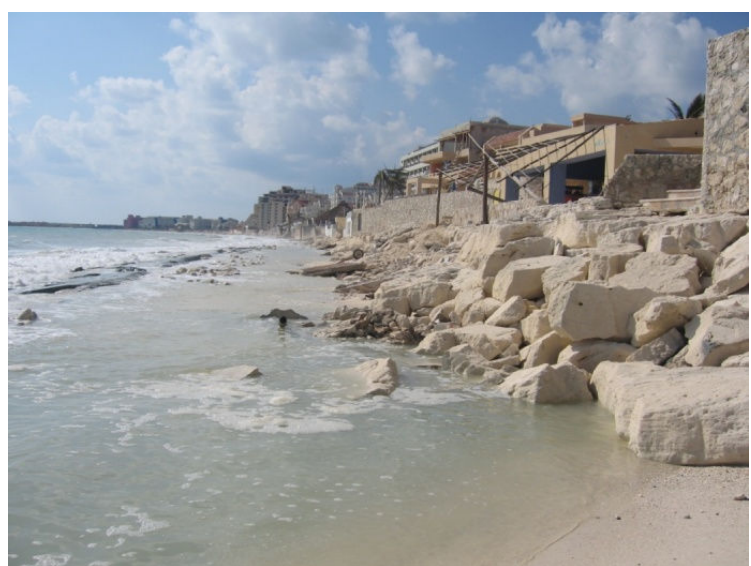

Figure 2. Example of beach erosion at Cancún, Mexico.

The work presented here focuses on testing and finding the optimal design of a tapered channel system by determining the geometric characteristics which would make energy conversion more efficient and thereby increase the possibilities of power generation. This paper presents the evaluation of two devices: a) a wave amplifier which, being overtopped, allows the storage of huge water volumes for further feeding a turbine and $b$ ) a new device designed to reproduce the wave amplification and air compression that occurs in natural structures known as "blowholes". This proposed system consists of a tapered pipe with an internal profile similar to that of a brass tuba. Its hydraulic operation goes from the wave steeping and concentration inside the device to the expulsion of an intermittent jet which can be directed to a turbine. The specific objectives of this experimental program, executed with both models, is to design a robust device for wave energy conversion based on the principle of wave amplification and/or ensnaring and to test and evaluate the efficiency of such a device in terms of energy conversion and protection.

\section{DEVICE DEVELOPMENT}

\section{Wave amplifier}

The first model tested consists of a narrowing channel similar to that of a tapchan. The structure has three main parts (see Figure 3): A) the wave amplifier, which catches the incident waves and ensures wave overtopping, being composed of a ramp and two movable vertical walls, B) the receiving structure, which is a vertical cylinder designed to reduce internal turbulence and oscillations, and C), the storage box, where the water is kept ready for power generation or further use.
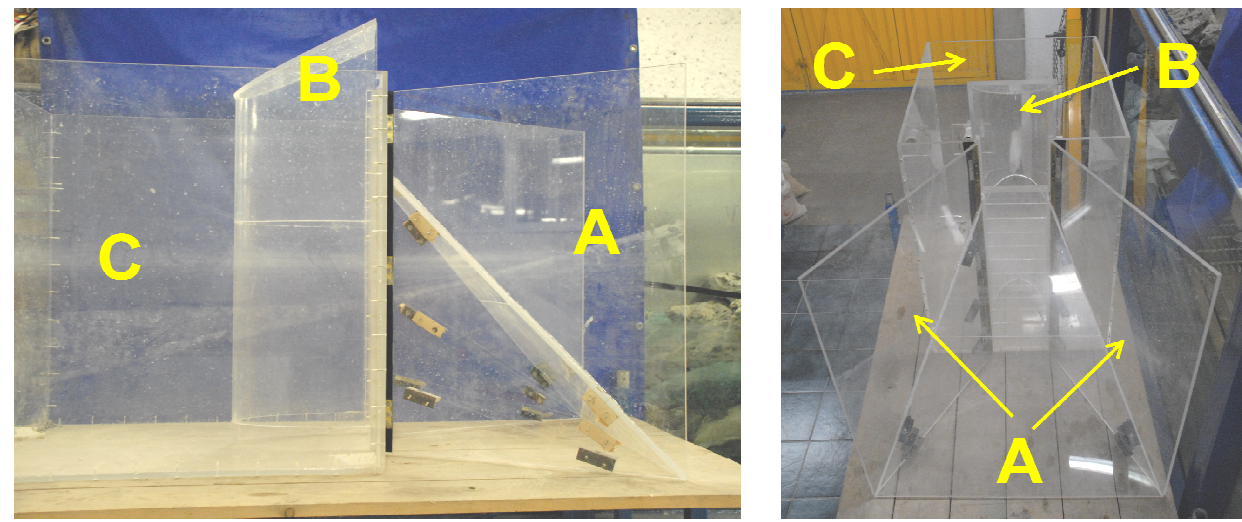

Figure 3. Laboratory model of the narrowing channel.

This structure is an adaptation of that proposed by Alatorre, 2003 with the difference that the latter was not intended to store water. The governing hydraulic phenomenon of the wave amplifier shown in 
Figure 3 is wave overtopping and the most important parameters for registering its behavior characterization are wave reflection and the average volume of water that can be stored with a given wave climate.

Thinking of this as a prototype, the fixed device has to be constructed onshore and needs huge land extensions in order to store enough water. It is certainly better to build these structures on coasts that will naturally favor its operation and increase its efficiency.

\section{Blow-Jet}

The second model is a novel proposal that takes some ideas from the tapchan and aims to reproduce the hydraulic behavior of the well known, but little studied, naturally occcurring blowhole structure. Blowholes occur all over the world and are known locally by different names; the most famous on the Mexican coast is that located on the North Pacific, in Ensenada, Baja California, and called the "Bufadora", which is an onomatopoeic term. Figure 4 shows two impressive examples of natural blowholes.

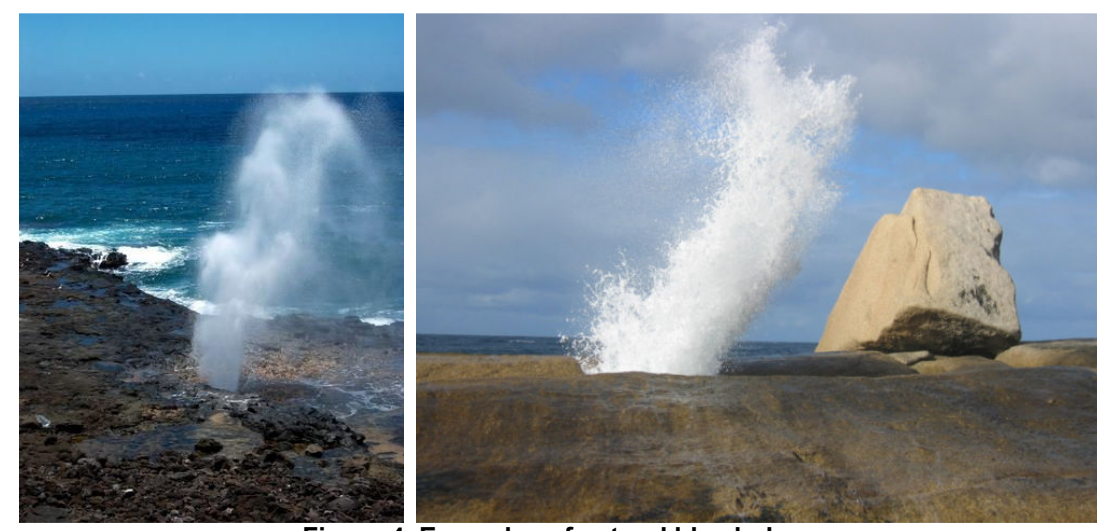

Figure 4. Examples of natural blowholes.

The complex internal morphology of blowholes has been poorly studied, what we do know is that this natural formation always presents a catchment entrance, a compression cavern and an expelling hole. The different sizes, slopes and arrangement of these three components determine the force and air-water mixture of the expelled jet. In terms of designing a wave energy converter based upon this hydraulic operation it is desirable to reduce wave reflection, minimize resonance inside the device and avoid excessive air-water mixture.

At the Engineering Institute of the UNAM three different small scale devices were constructed. The three have very similar geometries and are only differenced by the shape of the internal profile. The right hand image shown in Figure 5 corresponds to the device tested and presented in this paper, while that on the left presents the equations of the internal profiles for the three laboratory models.
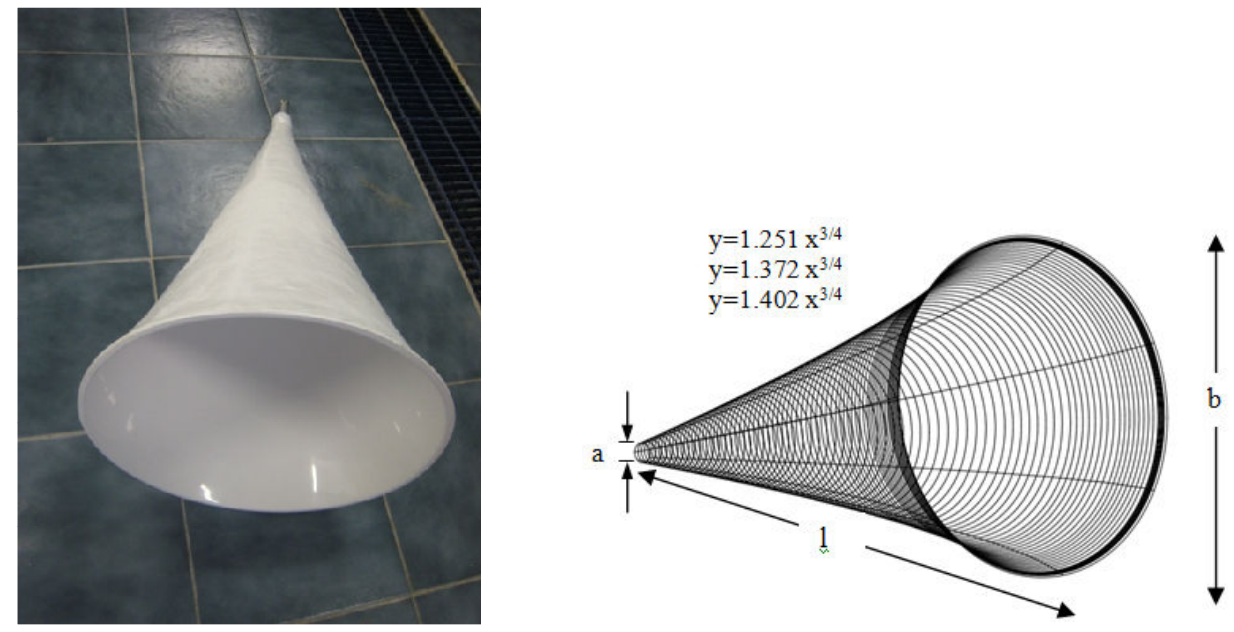

Figure 5. Blow-Jet laboratory model. 
The prototype of this model is considered as a fixed super-structure (i.e. it should be placed on top of another structure) whose main hydraulic function is the trapping and forcing of incident waves into a narrowing geometry where they are turned into a jet which can directly feed a simple turbine.

\section{LABORATORY TESTING}

All the experimental work was conducted at the wave flume of the National University of Mexico. This is a $37 \mathrm{~m}$ long, $1.2 \mathrm{~m}$ high and $0.8 \mathrm{~m}$ wide flume with a piston type wave maker. The bottom and ends of the flume are made of stainless steel and the two long sides are glass (Figure 6). The flume is equipped with an active wave absorber attached to the paddle at one end and a passive wave absorber at the other end.

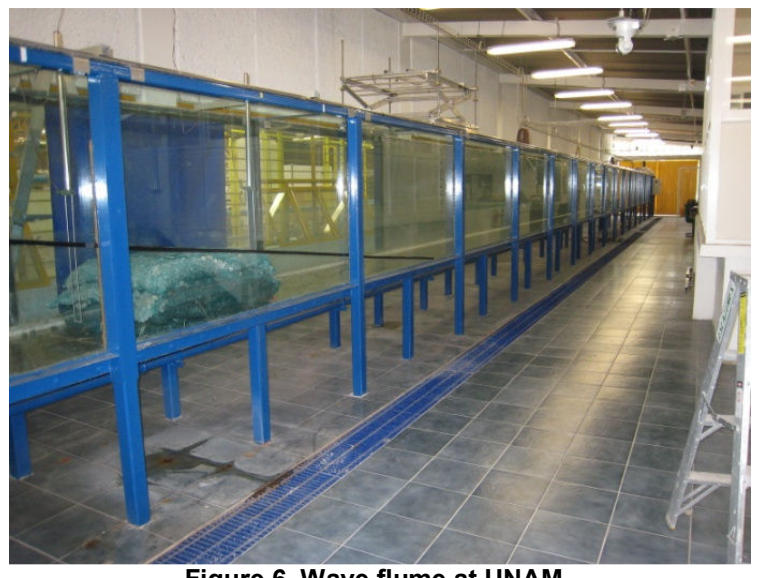

Figure 6. Wave flume at UNAM.

The necessities and operation of each of the two models described above was derived from the design of the two different experimental set-ups. The wave amplifier required a more complex arrangement of wave gauges in order to register the wave reflection generated by the ramp and walls of the narrowing channel. Another gauge was placed inside the storage box to register the water level. Schemes of the device in the wave flume are shown in Figures 7 and 8.

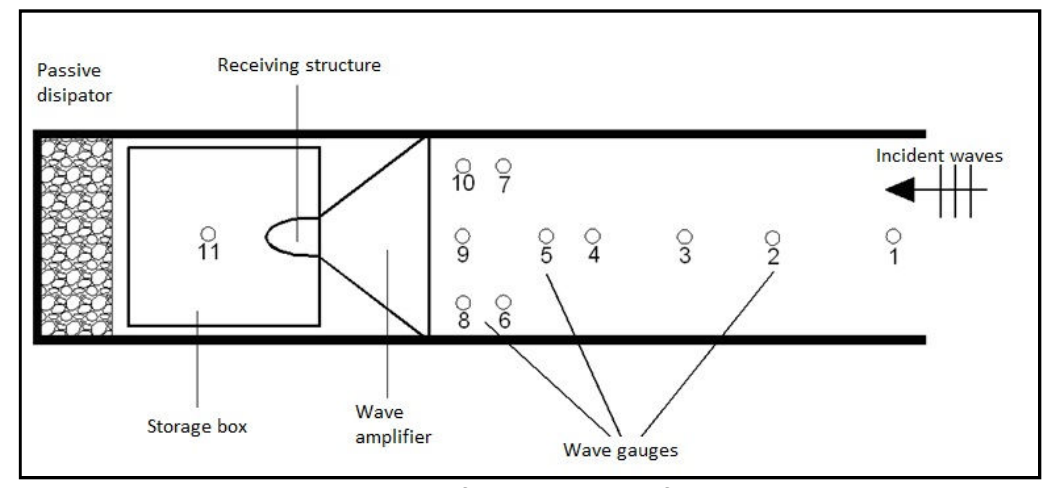

Figure 7. Plan of the wave amplifier set-up.

A total of 500 tests were carried out varying the angle of aperture of the vertical walls $\left(0\right.$ and $27.5^{\circ}$ from the flume axis), the ramp slope $\left(0,15^{\circ}, 30^{\circ}\right.$ and $45^{\circ}$ from the flat bottom), the wave period $(1.05$ to $2.0 \mathrm{~s}$ ) and wave height. The experimental procedure consisted of fixing an angle of the vertical walls, a ramp slope and a wave period and then generating regular wave trains starting with $0.15 \mathrm{~m}$ height and decreasing it a centimeter at a time until no overtopping occurred. The tests were made assuming that:

- The convergent vertical walls dramatically amplify the wave amplitude.

- The impermeable ramp ensures overtopping.

- The average volume of water per wave entering the storage box should depend on the combination of geometric and wave parameters.

- $\quad$ The device is fixed and the vibration due to wave impact is negligible. 
- The gravel beach below the structure (see Figure 8) reduces wave reflection and stops the generation of undesirable transversal waves.

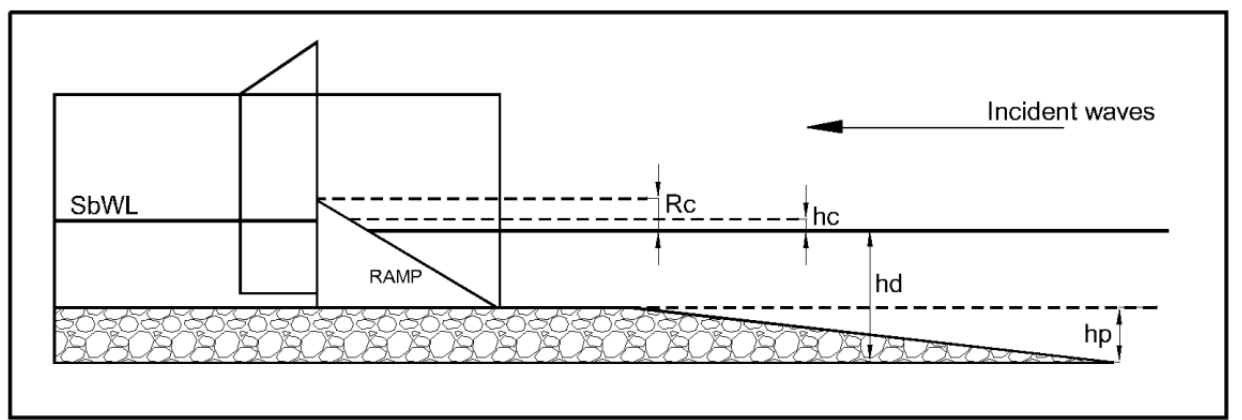

Figure 8. Lateral view of the wave amplifier experimental set-up.

In Figure $8 R c$ is the ramp freeboard, $h c$ is the vertical distance between the still water level and the water level inside the storage box (SbWL), $h d$ is the total water depth and $h p$ the thickness of the gravel support.

The experimental set up for the Blow-Jet consisted of three wave gauges at the front of the device for registering the incident and reflected waves and one more gauge behind the device to measure the transmitted waves. The water velocities at the entrance of the device and at the jet were measured with micro ADV's and PIV respectively (Figure 9).

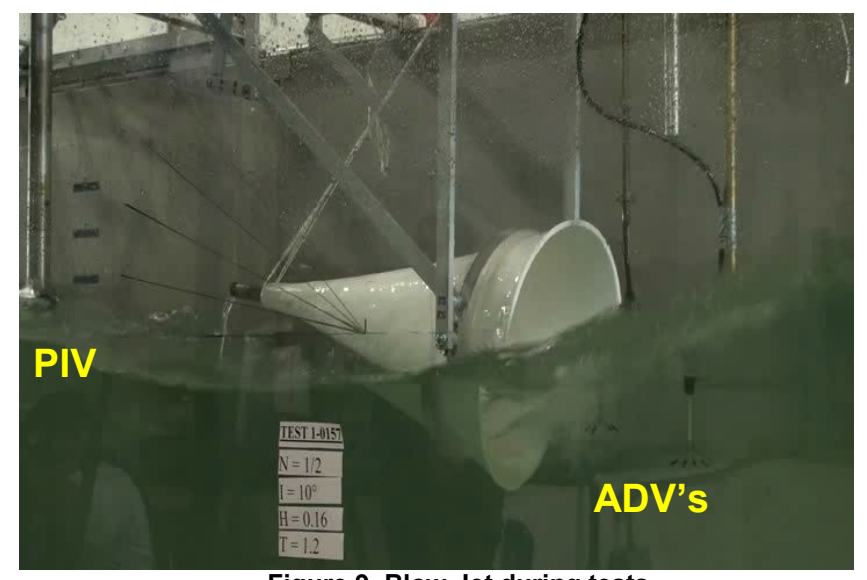

Figure 9. Blow-Jet during tests.

More than 300 tests were carried out with the Blow-Jet device; regular wave trains with different heights $(8$ to $20 \mathrm{~cm})$ and periods $(0.8$ to $2.0 \mathrm{~s})$ were generated. Four axis angles, from the still water level to the device were tested $(0,10,20$ and 30 degrees) for three relative submergences $(1 / 3,1 / 2$ and $2 / 3$ of the Blow-Jet entrance area). Some conditions were eliminated from the analysis because waves were breaking before reaching the device.

\section{RESULTS}

The data analysis presented in this paper focuses on evaluating the capacity and/or efficiency of the devices tested in terms of catching wave energy. That is, the first conclusion should be directed towards approving, or not, the device as a WEC. It is clear that in order to get to an eventual power generation plant scenario requires several other systems in addition, and each one has its own efficiency and study needs. The evaluation of the devices presented here is limited to stating the possibility of each model as the first step in the chain.

Similar importance is given to the analysis of the behavior of the devices as coastal protection alternatives. Together with the philosophy of taking energy from the sea, the need of coastal defenses can be satisfied if the new structures are planned as multi-propose infrastructure.

Evaluating the operation of the wave amplifier is quite simple; if the ramp is overtopped then some volume of water will be kept in the storage box. Then, for every combination of ramp angle, and 
vertical wall position there should be a wave climate with more average volume water per wave entering the box. The data collected during the experimental tests was divided in groups according to the value of $R$ and graphs were plotted showing the average discharge into the storage box, $Q$, versus $k h$. An example of these is shown in Figure 10.

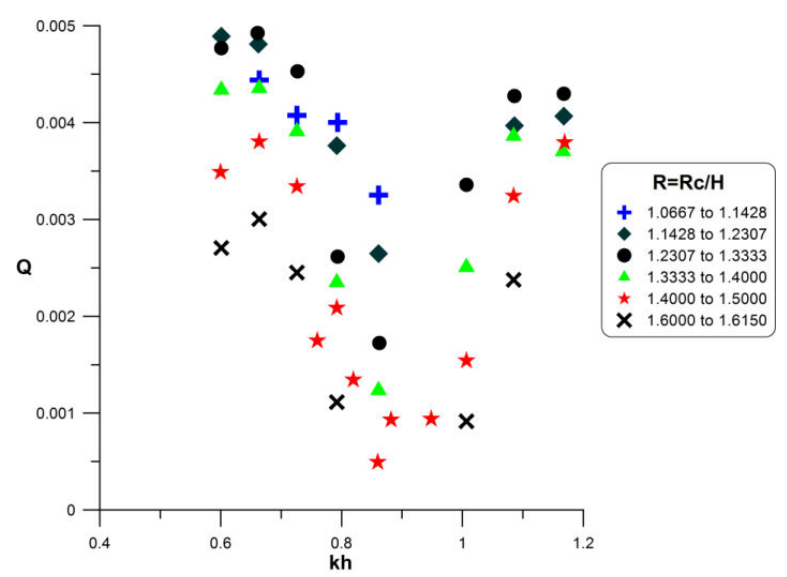

Figure 10. Discharge into the storage box as a function of wave climate for the $30^{\circ}$ ramp.

The trend of the plotted data, as seen in Figure 10, is parabolic in type. This tendency gives a very clear minimum discharge but also allows characterization of the range of $k h$ for higher discharge volumes. Table 1 is a summary of the best operation ranges of $k h$ for each angle of the ramp.

\begin{tabular}{|c|c|}
\hline $\begin{array}{c}\text { Table 1. Ranges of } \boldsymbol{k h} \text { for maximum discharge volume } \\
\text { entering the storage box for each ramp angle } \theta \text {. }\end{array}$ \\
\hline$\theta$ & $k h$ \\
\hline $15^{\circ}$ & $0.4-0.8$ \\
$30^{\circ}$ & $0.9-1.1$ \\
$45^{\circ}$ & $1.3-1.8$ \\
\hline
\end{tabular}

In Table 1 the data for $0^{\circ}$ (vertical wall) was eliminated because no overtopping occurred. It is also clear that as the angle of the ramp increases, the wave period for higher discharge volume is also higher. That is, for locations with small wave periods, the wave amplifier needs to have less steep slopes.

Data analysis found that the presence of the vertical walls, as part of the wave amplifier, directly affects the behavior of the device. Comparing the tests with no vertical walls and those with walls, the following linear relation could be defined

$$
\frac{Q_{1}}{l_{1}}=\alpha \frac{Q_{2}}{l_{2}}
$$

Where $Q_{1}$ and $Q_{2}$ are the average discharges for no walls and walls, respectively, $l_{1}$ is the receiving structure width and $\alpha$ is an amplifying coefficient of the discharge. Obviously, $\alpha$ being always greater than one means that the effect of the walls is to increase the average discharge. Table 2 shows the average values of the amplifying coefficient for each ramp angle tested in the laboratory.

\begin{tabular}{|c|c|}
\hline $\begin{array}{r}\text { Table 2. Average values of the amplifying coefficient } \\
\boldsymbol{\alpha} \text { for each ramp angle } \boldsymbol{\theta} \text {. }\end{array}$ \\
\hline$\theta$ & $k h$ \\
\hline $15^{\circ}$ & 1.86 \\
$30^{\circ}$ & 2.38 \\
$45^{\circ}$ & 2.71 \\
\hline
\end{tabular}

The wave amplifier tested in this work is a good alternative for catching wave energy and making it available for further use. One of the main advantages of this device is that as kinetic energy has been turned into potential energy, the possibility of keeping it stored until it is needed is real. The strength of 
this system is that once in the storage box, the oscillatory nature of the waves has been eliminated and it is easier to generate high quality power.

On the other hand, a great weakness of the wave amplifier is that in order to provide reasonable amounts of power, large land extensions are needed and it is clear that not all coasts are suitable because the construction of huge impermeable reservoirs for water would be very expensive and could be environmentally aggressive.

The evaluation of the wave amplifier as a coastal defense structure is not positive at all, the structure has to be attached to the coast and very poor or no protection to that coast can be provided. There is a possibility if the device is settled on top of a rubble mound, but then the distance between the receiving structure and the storage box can make the system useless. This leads us to conclude that although this structure can use waves as an energy source efficiently, it is not an alternative for coastal protection.

The evaluation of the Blow-Jet as a WEC was made in terms of the hydraulic efficiency of the device. The shape and internal profile of the Blow-Jet produces an important increase in the water velocity which can be measured at the entrance as the wave celerity and at the exit as the jet velocity. As was mentioned earlier, the velocity at the entrance was registered with acoustic means and at the exit with optic ones. Figure 11 shows a comparison between both velocities.

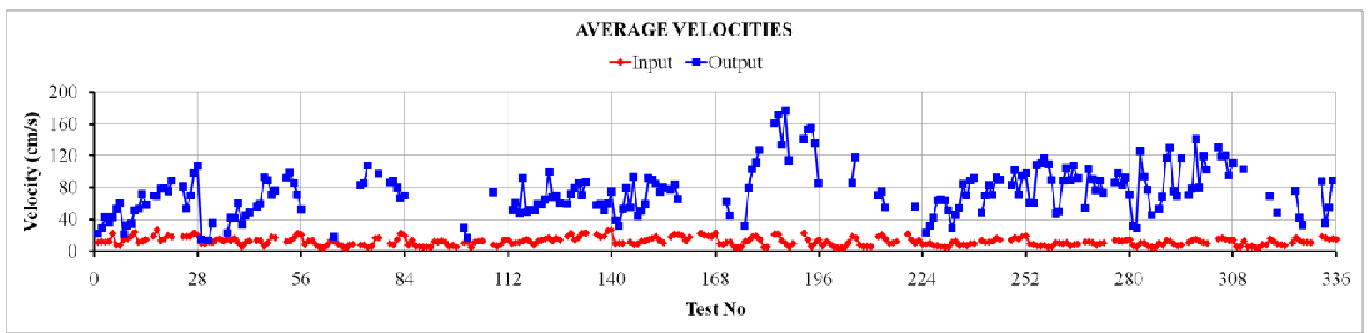

Figure 11. Velocity comparison at the entrance and exit of the Blow-Jet.

The input velocities in Figure 11 correspond to the average of the positive (wave propagation direction) velocities going into the Blow-Jet and the output ones to the average of the jet velocity while it was working as full pipe. The gaps in the output values correspond to the tests in which no jet could be generated. For simpler reference, in Figure 11 tests 1 to 112 correspond to $1 / 3$ of the entrance area submerged, 113 to 224 to $1 / 2$ and 225 to 336 to $2 / 3$. Each group has 16 tests corresponding to one axis angle $(0,10,20$ and 30 degrees) and within these the wave period and wave height was increased with the number of the test.

A simple comparison shows that almost all the tests gave velocity amplification. Figure 12 presents the ratio between the output and input velocities for each laboratory test. The red line shows the threshold of Vo, the output velocity, equal to $\mathrm{Vi}$, the input velocity.

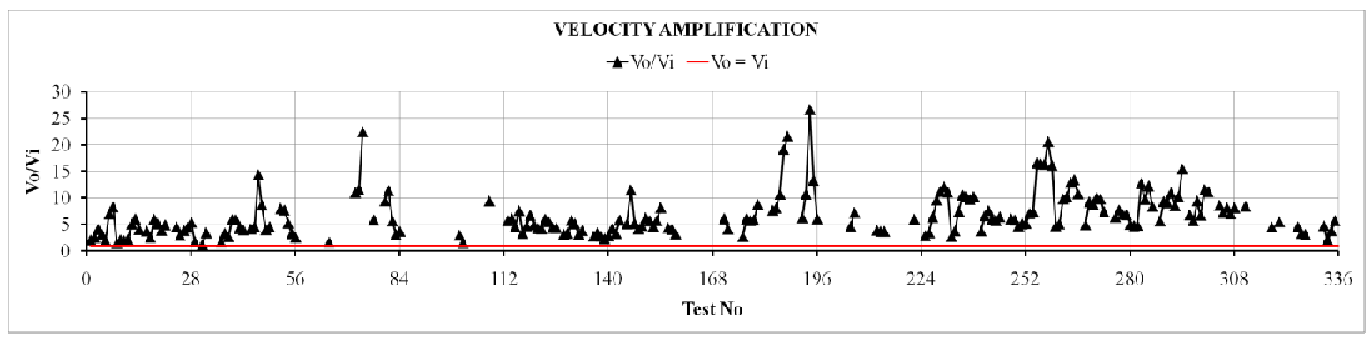

Figure 12. Output to input velocity ratio for Blow-Jet device.

From Figures 11 and 12 it is clear that the highest amplification of velocity occurs when the area of the entrance of the Blow-Jet is half submerged and the axis angle is $20^{\circ}$ (test 196). The configuration with best overall performance however is where $2 / 3$ of the entrance area was submerged and the axis angle was $0^{\circ}$ to $10^{\circ}$. In turn the worst performance was with $1 / 3$ of the entrance area submerged and the axis angle being $20^{\circ}$ to $30^{\circ}$. Here the ratio is below one (less velocity at the exit than at the entrance) and in several tests no jet at all was produced. 
Although several tests have been carried out, it is not yet easy to characterize the behavior of the device as only one geometric form has been tested. The other two internal profiles are still being analyzed in the laboratory. Even so, with the available data it is possible to get some information on how the Blow-Jet is working hydraulically. This information will also help in the development of complementary experimental tests.

One of the first relations found during the analysis is that the operation of the Blow-Jet has a strong dependency on $k h$, that is, for some of the data it is clear that the output velocity increases with $k h$. This is shown in Figure 13.
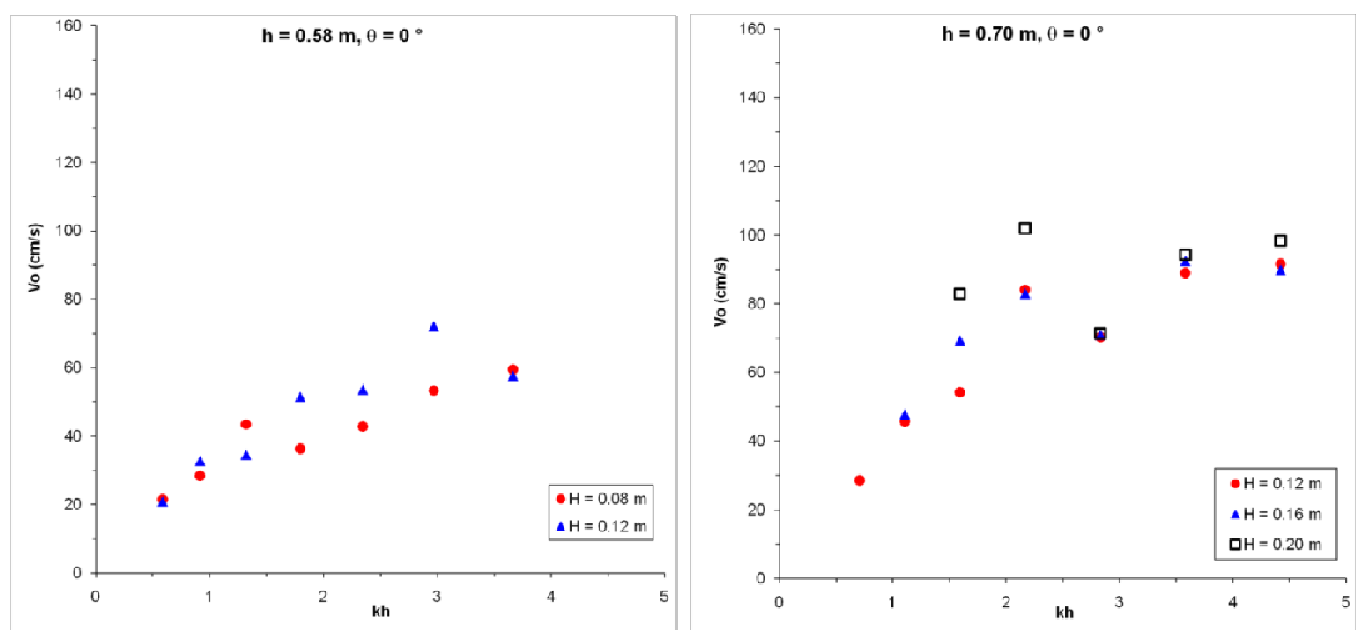

Figure 13. Groups of tests showing the dependency of Vo on kh.

The left hand panel of Figure 13 shows the tests corresponding to a submergence of $1 / 3$ of the entrance area and the axis of the Blow-Jet being totally horizontal; the right hand panel corresponds to $2 / 3$ of the entrance area being submerged and the axis angle being horizontal again. In both cases a quasi linear relation between Vo and $k h$ can be seen.

Another effect seen frequently in the experimental data is the presence of a maximum value of Vo for some values of $k h$. Figure 14 presents two specific cases in which the maximum velocity occurs around $k h=2$. It is remarkable that in the right hand panel of Figure 13 the same maximum is found and also near the same value of $k h$.
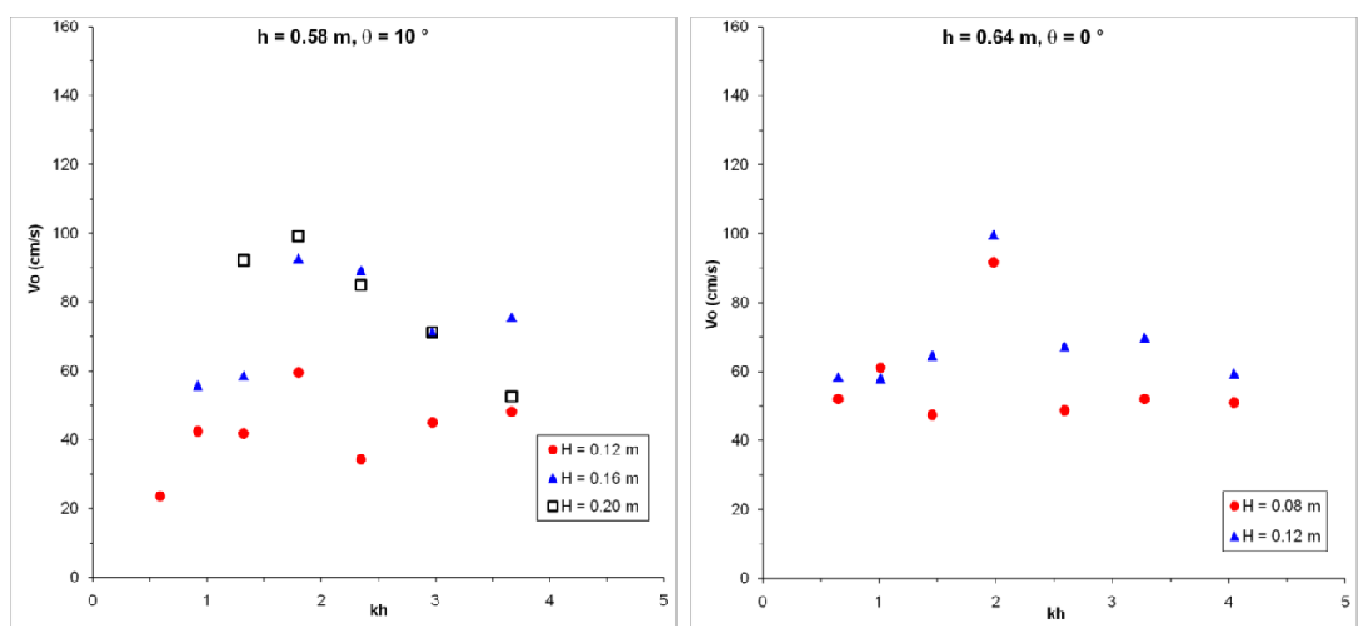

Figure 14. Groups of tests showing the occurrence of a maximum in Vo for $k h$ value close to 2.0 .

The existence of this maximum value of Vo is a good argument to accept the Blow-Jet as a WEC, because not only is the hydraulic efficiency reasonable but also the behavior of the device can be represented as a function of the wave climate $(k h)$ and for some cases the geometry can also be 
specifically determined for local wave conditions. This can be understood as the first step on the way to finding design recommendations and, eventually, equations for a design, depending on the energy available locally. In simple words, the size of the Blow-Jet is a direct function of the wave period.

The evaluation of the device as an alternative for coastal protection was made by reviewing the transmitted energy, taken as the energy that can be measured past the Blow-Jet. Knowing the incident wave height (separating the incident and reflected waves as Baquerizo, 1995) and the waves measured after the jet, a transmission coefficient was estimated with

$$
|T|=\frac{H_{T}}{H_{i}}
$$

where the subscripts $T$ and $i$ stand for the transmitted and incident wave heights, respectively and $|T|$ is the transmission coefficient. The transmission coefficient was plotted versus the total wave height, $\mathrm{H}$, relative to the diameter of the Blow-Jet entrance, 1 . The plots are presented in Figures 15 and 16.
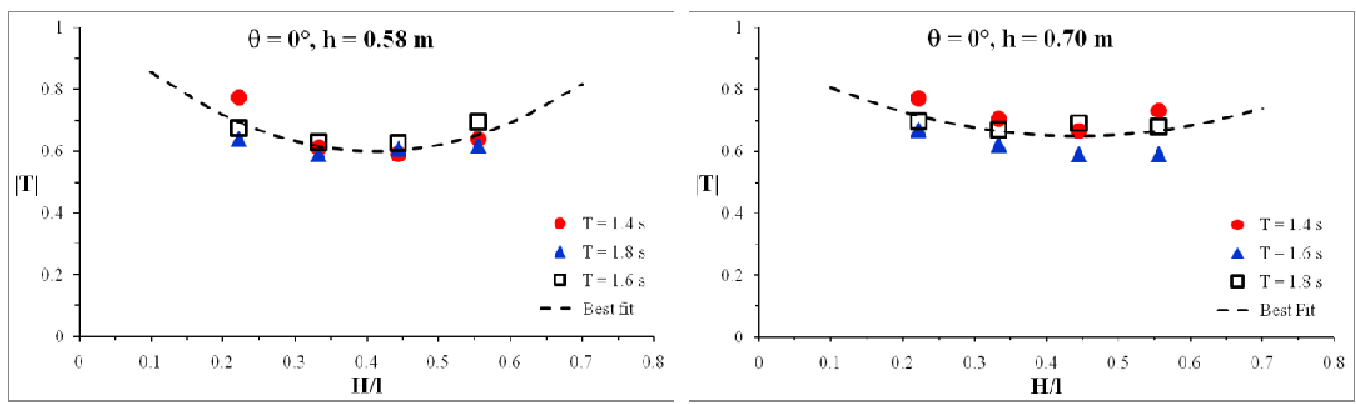

Figure 15. Transmission coefficient for Blow-Jet horizontal axis.
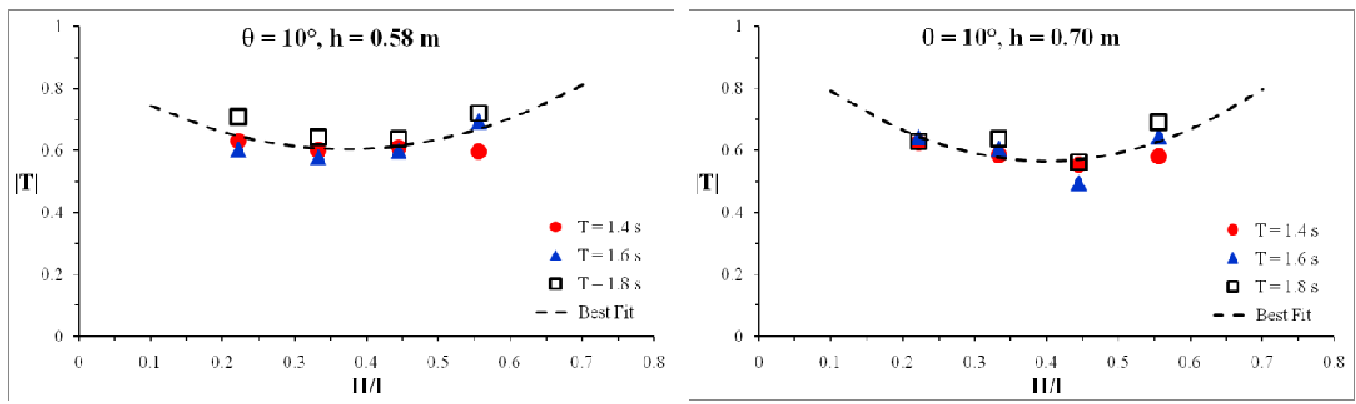

Figure 16. Transmission coefficient for Blow-Jet axis at $10^{\circ}$.

The parabolic relation shown in Figures 15 and 16 with a minimum value for transmission close to $\mathrm{H} / \mathrm{l}=0.4$ leads us to conclude that the design of the Blow-Jet for specific conditions can be optimized for very little transmission and as the value of the coefficient can be very close to 0.5 its efficiency as a shore protection structure is quite good.

It has to be said that the Blow-Jet development is not finished; the way in which it would be implemented in the field is not still defined. Some ideas are to construct it as a floating structure in which case the transmission should be as presented in this paper. The second idea is to lay it on top of a rubble mound which will reduce the transmission furthermore. In any case this first analysis puts the Blow-Jet in the list of multi-purpose structure alternatives.

\section{CONCLUSIONS}

Wave energy conversion combined with shore protection is feasible, the hydraulic and energy dissipation of the devices must be studied further but the results obtained here are encouraging.

Tapchan WECs are simple to design and construct but their major disadvantage is the need for huge water reservoirs. They are also a limited alternative for coastal protection, but a very useful possibility when the natural morphology favors their construction. 
Further work is needed to find optimum geometries for the Blow-Jet device for given wave climate conditions. The parameters that can still be improved are the shape and position of the compression camera, the shape of the internal profile and the possibility of leaving part of the device at the free surface in order to reduce wave reflection and forcing more energy to feed the expelled jet.

Finding the geometry of minimum transmission with maximum energy conversion efficiency as well as determining the best way to implement the Blow-Jet in field are the major tasks for further work.

\section{ACKNOWLEDGMENTS}

"The support of the European Commission through FP7.2009-1, Contract 244104 - THESEUS ("Innovative technologies for safer European coasts in a changing climate"), is gratefully acknowledged.

\section{REFERENCES}

Alatorre, M. 2003. Sea water pumping system using wave and tide energies. PhD Thesis. UNAM, Mexico. 201 pp. (In Spanish).

Baquerizo A. 1995. Wave reflection at beaches. Prediction and evaluation methods. $\mathrm{PhD}$ Thesis, University of Cantabria, Santander, Spain. 180 pp. (In Spanish).

Hsu, J., Klein, A., Benedict, L. Raabe, A. and Tsai, C. 2005. Static equilibrium bay beach for coastal management and protection. Seminar Series, University of Sydney. 52 pp.

IEA-OEs (2008) Annual Report, International Energy Agency Implementing Agreement on Ocean Energy Systems (IEA-OES), edited by A. Brito-Melo and G. Bhuyan, February 2009.

Leijon M., Danielsson O., Eriksson M., Thornburn K., Bernhoff M., Isberg J., Sundberg J., Ivanova I., Sjöstedt E., Agren O., Karlsson K. and Wolfbrandt, A. (2006) An electrical approach to wave energy conversion. Renewable Energy. Vol. 31:9, pp. 1309-1319.

Uriarte, A. (2003). Historia del Clima en la Tierra. Servicio Central de Publicaciones del Gobierno Vasco. 306 pp. 